\title{
DESIGN AND FABRICATION OF INTEGRATED PIEZOELECTRIC MICROPUMP WITH VORTEX ENHANCEMENT OPTIMIZATION
}

\author{
Boshen Liang ${ }^{12}$, Louis Paquet ${ }^{1}$, Yongbin Jeong ${ }^{12}$, Paul Heremans ${ }^{12}$, and David Cheyns ${ }^{1}$ \\ ${ }^{1}$ IMEC, Leuven, BELGIUM \\ ${ }^{2}$ KU Leuven, Leuven, BELGIUM
}

\begin{abstract}
In this study, a 3D acoustic-solid interaction model is combined with a time-dependent vorticity enhancement method for design optimization of nozzle/diffuser micropumps. Compared with conventional method where static pressure loss ratio is used as the performance indicator of individual nozzle/diffuser element, the vortex method provides direct and good prediction of output characteristics of the micropumps.

An integrated piezoelectric micropump prototype is fabricated with flat-panel display compatible processing techniques. The frequency characterization shows good agreement of the micropump working frequency with that predicted by finite element simulation where additional damping effects are considered. A high-speed particle image velocimetry shows successfully the movement of fluid element driven by the piezoelectric actuator.
\end{abstract}

\section{KEYWORDS}

Micropump, Nozzle/Diffuser, Lab-on-Chip, Vorticity, Optimization

\section{INTRODUCTION}

Micropumps are one of the indispensable components for integrated Lab-on-Chip (LOC) devices [1]. Among different concepts, nozzle/diffuser micropumps attract scientific attention since its flow regulation function is achieved with non-moving geometrical structures, which decrease dramatically the risk of issues such as channel clogging, fluid leakage and thus device failure [2].

Similar to diodes for electronics, the effectiveness of a diffuser for flow directing can be defined as Diodicity [3]. However, following the conventional method, the nozzle/diffuser elements with larger diodicity calculated based on static fluidic simulation fail to achieve higher flowrate for micropumps. This may be caused by the pulsatile nature of the flow when micropumps are driven by periodic forces, which existing models do not take into account.

In this study, a 3D acoustic-solid interaction model is used to set the working frequency range, and then the proposed time-dependent vortex-based enhancement is used for design optimization. A prototype device is fabricated following the optimization result, and frequency and flowrate characterization are conducted.

\section{MODELLING}

A schematic of the micropump is shown in Fig.1. In our design, a $30 \mu \mathrm{m}$ deep microfluidic channel is covered with $15 \mu \mathrm{m}$ polyimide (PI) membrane, which is driven by a micromachined 500nm thick piezoelectric (polyvinylidene difluoride, PVDF) transducer unit [4] to provide periodic force into the fluid system. The working fluid used for this work is water, which is featured with much higher inertial effect compared to the case when air is used as working fluid. Two nozzle/diffuser valve units are added to the left and right side of the central chamber for modulation of the net flow direction.

In this study, the central circular chamber radius is constant $(450 \mu \mathrm{m})$, while several geometrical diffuser parameters shown in Fig.1(b) are optimized to get the best micropump performance, i.e. the highest flowrate. In the model, $D_{\text {in }}$ and $D_{\text {out }}$ are the inlet and outlet width, $\alpha$ is the opening angle, $L$ is the length, $H_{c h}$ is the connecting fluid channel height, and $R$ is the corner fillet radius.

\section{Frequency response}

One of the major features of micropumps driven by periodic forces is that the flow inside the fluid system is pulsatile. Analytically, for a microfluidic channel with circular cross-sections, providing there is no flow rotation and the flow is fully developed, the Navier-Stoke equation in polar coordinate system can be simplified to

(a)

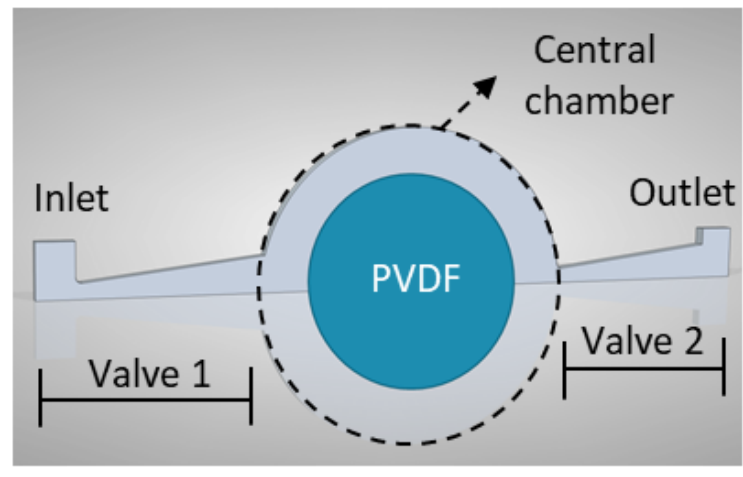

(b)

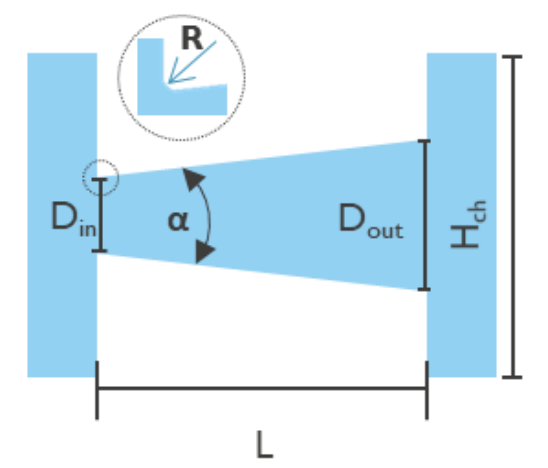

Figure 1: Schematics of diffuser/nozzle micropump driven by a piezoelectric actuator (a) Configuration of the micropump with a central chamber connected by two diffuser/nozzle elements as flow rectifying diodes. (b) Design parameters of a diffuser/nozzle valve unit. 


$$
\frac{\partial P(x, t)}{\partial x}=\mu\left(\frac{\partial^{2} u(r, t)}{\partial r^{2}}+\frac{1}{r} \frac{\partial u(r, t)}{\partial r}\right)-\rho \frac{\partial u(r, t)}{\partial t}
$$

where $P$ is the periodic input force expressed as pressure term, $\mu$ and $\rho$ are the viscosity and density of water, $x$ and $r$ are the longitudinal and polar axis, $u$ is the velocity of fluid, and $t$ is the time.

Following similar approach introduced in previous research [5], by assuming the pressure term to the left side of eqn (1) is periodic with the form $P_{s} e^{i \omega t}$, the fluid velocity $u(r, t)$ in the channel can be expressed to have the form

$$
u_{\emptyset}(r, t)=\frac{i P_{S} R^{2}}{\mu W^{2}} \times\left(1-\frac{J_{0}\left(i^{3 / 2} W \frac{r}{R}\right)}{J_{0}\left(i^{3 / 2} W\right)}\right) \times e^{i \omega t}
$$

where $P_{S}$ is the pressure amplitude, $R$ is the radius of the channel, $J_{0}$ is the order 0 Bessel function of first kind, and $W$ is the Womersley number with the form $W=$ $\mathrm{R} \sqrt{\rho \omega / \mu}$. Here $\omega$ is the angular frequency of the external periodic driving force. The three terms in eqn (2) represents the amplitude factor, shape factor and periodic term of the pulsatile flow velocity profiles, respectively.

The solution of eqn (2) is quantitatively shown in Fig.2. It shows that with the increase of driving frequency and thus the Womersley number $\alpha$, the fully developed flow profile changes from a parabolic shape to a flatter shape, while the velocity amplitude of the fluid decreases dramatically. The nozzle/diffuser elements will eventually lose its fluid directing ability at extremely high frequency range since the inertial effect of the working fluid stop itself from moving at any meaningful velocity.
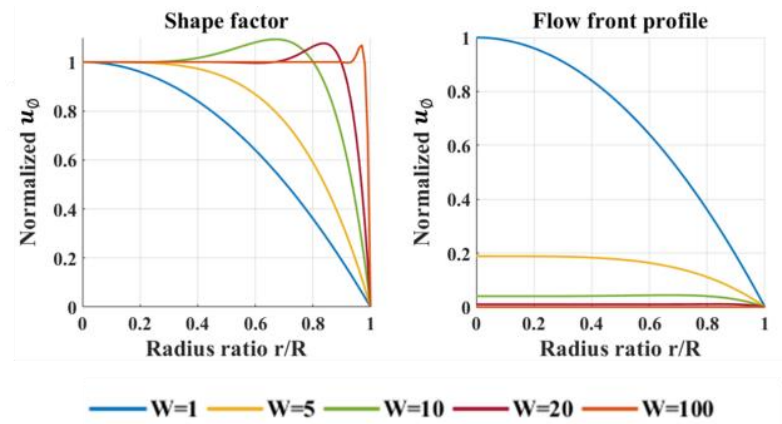

Figure 2: Solution of simplified Navier-Stroke equation. The shape factor and flow front profile are graphically plotted for different Womersley numbers.

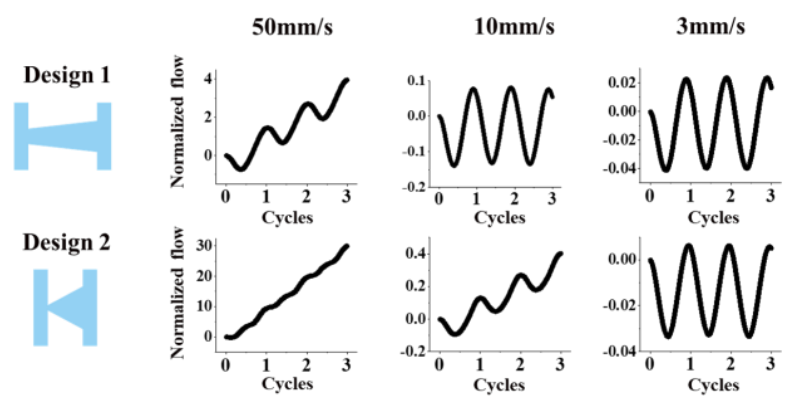

Figure 3: Comparison of full micropump flowrate results for two distinct nozzle/diffuser designs with same $D_{i}$ values, which fails to predict the apparent higher performance of Design 2 compared to Design 1.
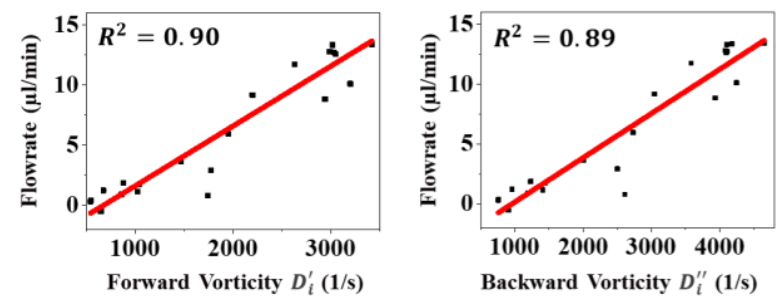

Figure 4: Relationship of micropump flowrate with forward/reverse vorticity of diffuser/nozzle element. A good linear regression indicates the vorticity can be used as a good performance indicator of micropump output performance.

A 3D acoustic-solid interaction model is built up in COMSOL to calculate the resonant frequency of the vibrating membrane. Compared with the analytical Rayleigh-Ritz method [11-12] which is only capable of calculating the resonance frequency of a circular membrane in contact with fluid half space, the COMSOL model takes into account extra damping effects imposed by shallow microfluidic channel and diffuser/nozzle elements. Based on the fixed chamber radius and chamber depth used in this study, the simulated $1^{\text {st }}$ mode resonant frequency of the PI membrane with water as working fluid is in the range of $3 \mathrm{kHz}-5 \mathrm{kHz}$. Eqn (2) reveals that this frequency range wouldn't degrade the nozzle/diffuser performance towards non-working zones while it can ensure the maximum vibrating amplitude.

\section{Vortex-based optimization method}

A better diffuser design is figured with higher ability of allowing forward flow while inhibiting reverse flow. Conventionally, the ratio of the pressure drops in backward direction to that in forward direction is named as Diodicity:

$$
D_{i}=\frac{\Delta P_{\text {backward }}}{\Delta P_{\text {forward }}}
$$

However, this definition fails to take into account the transient of the pulsatile flow inside micropumps driven by periodic forces. As an example shown in Fig.3, two distinct diffuser designs with same $D_{i}$ as 1.35 are plugged into full 3D micropump model to calculate flowrates at three different membrane vibrating peak velocities. Apparently, the pressure drop ratio from static simulations cannot predict well the micropump performance difference.

To overcome this discrepancy between static diffuser performance indicator and micropump performance, two time-dependent parameters are proposed as

$$
\begin{aligned}
D_{i}^{\prime} & =\frac{\int_{0}^{T / 2} V_{\text {forward }} d t}{T / 2} \\
D_{i}^{\prime \prime} & =\frac{\int_{T / 2}^{T} V_{\text {backward }} d t}{T / 2}
\end{aligned}
$$

where $V$ is the vorticity with the unit $1 /$ second, and $T$ is the period of a full pumping cycle obtained from previous frequency response analysis. Despite the same $D_{i}$ values for the two diffuser designs in Fig.3, the $D_{i}^{\prime}$ and $D_{i}^{\prime \prime}$ values are different and correspond well to the different micropump performance. Furthermore, 22 extra diffuser designs with a combination of different diffuser/nozzle parameters are simulated. As shown in Fig.4, a good linear 
1. PI spinning and peeling

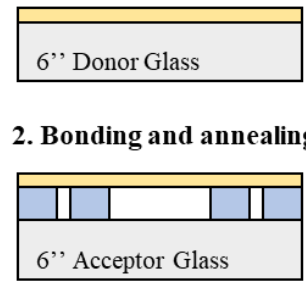

3. Bottom contact pattering

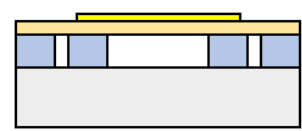

4. PVDF spinning and baking
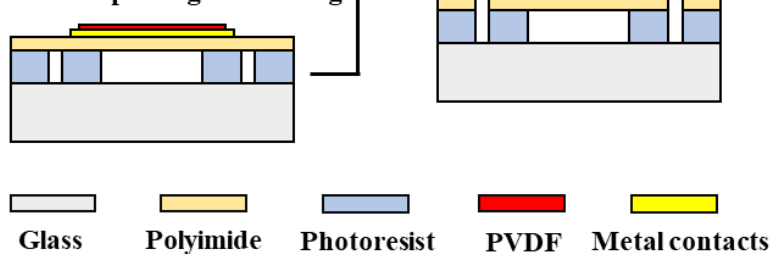

5. Top contact patterning

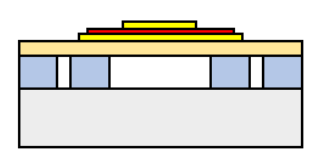

6. PVDF polling

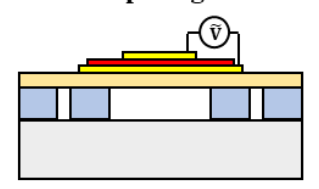

7. Laser ablation of PI

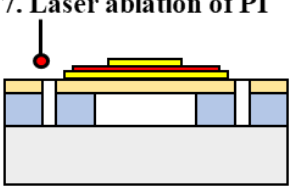

PVDF Metal contacts

Figure 5: A flat-panel display compatible fabrication process of the micropump.

relationship can be established, which indicates that the two parameters from time-dependent simulation can be used as a good performance indicator for the micropump.

\section{FABRICATION}

The fabrication process is schematically shown in Fig.5. Firstly, a polyimide resin was spin-coated on 6-inch a donor glass wafer, and then fully cured in $\mathrm{N}_{2}$ atmosphere at $300{ }^{\circ} \mathrm{C}$ for 3 hours. The $15 \mu \mathrm{m}$ PI membrane was then peeled off from the donor glass substrate and applied to the acceptor wafer where a $30 \mu \mathrm{m}$ photoresist was spin-coated and patterned on top by standard lithography step to form the microfluidic channel. A bonding and annealing in vacuum were followed to achieve permanent bonding between PI and microfluidic channel. Then, $60 \mathrm{~nm}$ aluminum was deposited and patterned on top of PI as bottom contact. P(VDF-TrFE) powder was dissolved in Methyl Ethyl Ketone and the solution was spin-coated on top of the wafer to form a $0.5 \mu \mathrm{m}$ piezoelectric layer. The PVDF layer was cured in $\mathrm{N}_{2}$ atmosphere at $140{ }^{\circ} \mathrm{C}$ for 1 hour. Another $60 \mathrm{~nm}$ aluminum layer was deposited and patterned as top metal contact. In this study, the metal coverage ratio is set to $67 \%$ based on a previous study [8] to have the optimal driving efficiency.

The PVDF layer was polarized by applying $80 \mathrm{~V}$ peakto-peak triangular waves at $100 \mathrm{~Hz}$ for 200 pulses. Finally, the inlet and outlet of the microfluidic chip were opened by laser ablation of the PI.

\section{EXPERIMENTS \& RESULTS}

A prototype device is successfully manufactured based on the above optimization and fabrication methods. Each micropump unit occupies less than $1.5 \mathrm{~mm}$ x $1 \mathrm{~mm}$ area on glass wafer. A function generator and a voltage amplifier were used to apply square waveform signal to drive the PVDF membrane. The applied peak-to-peak voltage $\left(\mathrm{V}_{\mathrm{pp}}\right)$ amplitude was $1 \mathrm{~V}$ for vibration

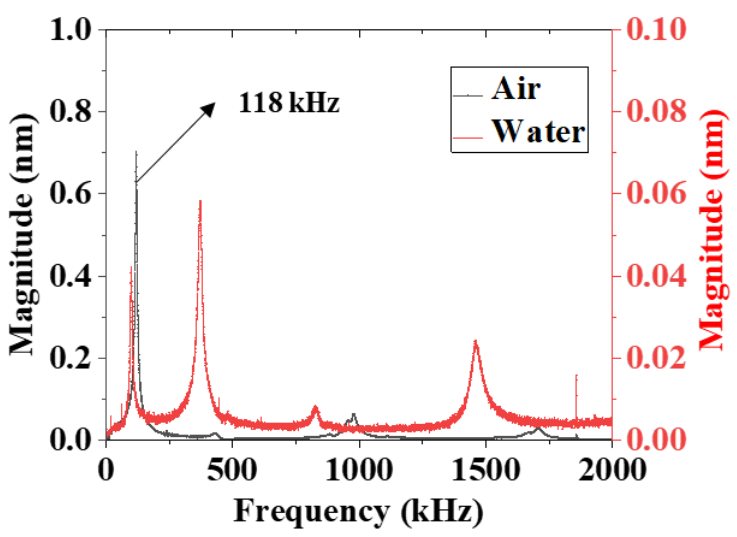

Figure 6: Frequency response of the fabricated micropump with or without fluid channel filled with water. This result is measured by LDV high frequency unit. Magnitude is measured at $V_{p p}=1 \mathrm{~V}$.

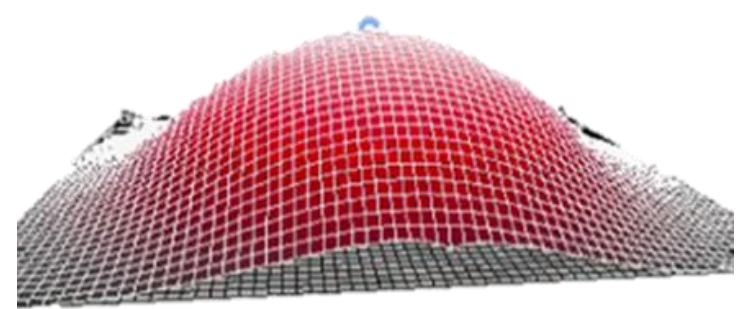

$200 \mu \mathrm{m}$

Figure 7: $3 D$ reconstructed shape of the vibrating membrane when the fluid channel is filled with water. This result is measured by $L D V$ low frequency unit at unit voltage.

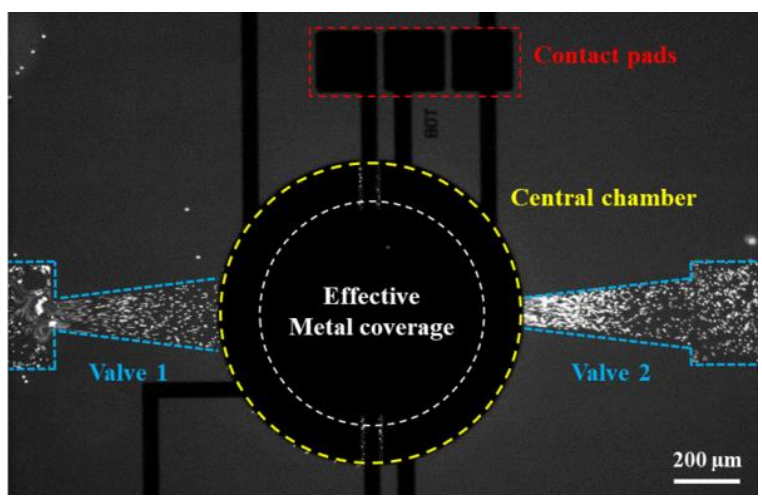

Figure 8: MicroPIV test image. The florescent microspheres aggregate to the right side of the micropump, which illustrates a net flow from left to right side.

characterization and $80 \mathrm{~V}$ for flowrate characterization.

Laser Doppler vibrometer (LDV, Polytec MSA500) was used for membrane vibration characterization. Firstly, a high frequency unit was used to measure the vibration of the PI membrane with and without the channel filled with water. As seen in Fig.6, as a consequence of additional damping imposed by introducing water into the microfluidic channel, both the resonant frequency peaks 

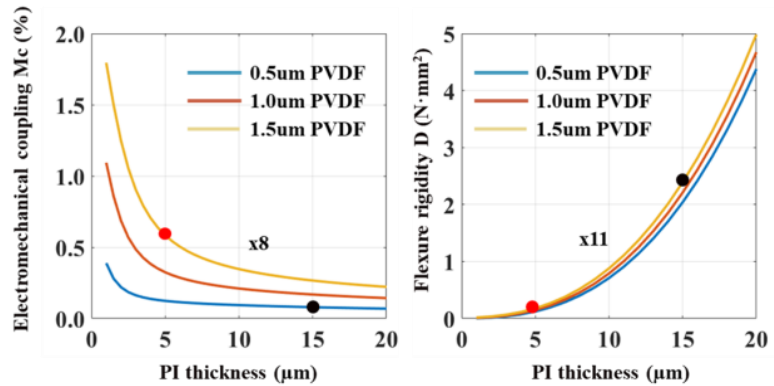

Figure 9: Impact of different PVDF and PI thickness on the micropump vibrating amplitude. Black dots are current thickness combination, and red dots are proposed new combination for higher flowrate.

and amplitudes changed after the membrane is contacted with water. For air, the resonance peak at $118 \mathrm{kHz}$ corresponds to the 1 st mode vibration. In order to capture the $1^{\text {st }}$ mode in water, the LDV was switched to low frequency measurements. In Fig. 7 , the $1^{\text {st }}$ mode vibration of the membrane at $4.4 \mathrm{kHz}$ is reconstructed in 3D form. The measured frequency matches well with the frequency range predicted by $3 \mathrm{D}$ acoustic-solid interaction model.

Micron resolution particle image velocimetry (MicroPIV) was used for micropump flowrate characterization. Carboxylate-modified microspheres (FluoSpheres ${ }^{\mathrm{TM}}, 2.0$ $\mu \mathrm{m}$ diameter, $515 \mathrm{~nm}$ fluorescence peak) was diluted in water and then injected in the fluid channel, and an inverted microscope setup with a high-speed camera was used to capture the movement of the microspheres. As shown in Fig.8, after turning on the driving voltage, the florescent microspheres start aggregating towards the right side of the micropump, which illustrates a net flow from left to right side. Driven by periodic square voltage input at $80 \mathrm{~V}_{\mathrm{pp}}$, the PI membrane vibrated at the velocity of $3 \mathrm{~mm} / \mathrm{s}$, and the overall net flowrate was estimated to be around $20 \mathrm{nl} / \mathrm{min}$ from particle tracking result.

The achievable flowrate with current technology can be easily increased to $\mu \mathrm{l} / \mathrm{min}$ range. From the $1^{\text {st }}$ mode frequency drop from $118 \mathrm{kHz}$ in air to $4.4 \mathrm{kHz}$ in water, it can be deduced that the vibrating membrane is in near overdamping status. Based on the simplified 1D spring-mass theory, the vibrating amplitude of the piezo-driven membrane $w$ can be modeled as

$$
w=\frac{F_{0}}{k} \sim \frac{E \times D_{31} \times M_{c}}{D}
$$

where $F_{0}$ is external force amplitude, $k$ the equivalent spring constant of the multi-layer membrane system, $E$ is the electric field applied to PVDF, $D_{31}$ is the PVDF piezoelectricity constant, $M_{c}$ is the electromechanical coupling efficiency [9], and $D$ is the flexural rigidity of the multi-layer membrane system. For a larger membrane vibrating amplitude and hence a higher micropump flowrate, apart from increasing electric field $E$ or replacing PVDF with other piezoelectric materials for higher $D_{31}$, changing the thickness of PVDF and PI can be two other effective choices. As illustrated in Fig. 9, a combination of thinner PI and thicker PVDF layer could boost the vibrating amplitude and thus the flowrate by $10-100$ times via enhancing the electromechanical coupling efficiency and decreasing the equivalent system spring constant.

\section{CONCLUSION}

We have developed an efficient and effective method for nozzle/diffuser micropump optimization by combining a 3D acoustic-solid interaction model with a timedependent vorticity enhancement method. It overcomes the drawback of conventional static optimization method where the transient feature of pulsatile flow for micropumps driven by periodic force are omitted. The fabrication method of our prototype device is featured with superior compatibility to flat-panel display manufacturing process, and it can be easily extended to cover huge flowrate range from $\mathrm{nl} / \mathrm{min}$ range to $\mu \mathrm{l} / \mathrm{min}$ range. This guarantees the proposed device is not restricted in labs, but has the potential for mass production in fabs in mature phase.

\section{ACKNOWLEDGEMENTS}

We greatly appreciate the contributions and meaningful discussion from several members of imec Large Area Electronics team.

\section{REFERENCES}

[1] P. Woias, "Micropumps - past, progress and future prospects," Sensors Actuators B Chem., vol. 105, no. 1, pp. 28-38, Mar. 2005.

[2] A. Olsson, P. Enoksson, G. Stemme, and E. Stemme, "Micromachined flat-walled valveless diffuser pumps," J. Microelectromechanical Syst., vol. 6, no. 2, pp. 161-166, Jun. 1997.

[3] A. R. Gamboa, C. J. Morris, and F. K. Forster, "Improvements in fixed-valve micropump performance through shape optimization of valves," $J$. Fluids Eng. Trans. ASME, vol. 127, no. 2, pp. 339346, Mar. 2005.

[4] Y. Jeong, C. Huang, D. Cheyns, and G. B. Torri, "pMUT device compatible with large-area display technology," 16th Int. Symp. Electrets, p. 2004, 2016.

[5] M. Zamir, "Basic Elements of Pulsatile Flow," Springer, Cham, 2016, pp. 81-122.

[6] M. Kwak, "Vibration of circular plates in contact with water," 1991.

[7] M. Kwak, K. K.-J. of S. and Vibration, and undefined 1991, "Axisymmetric vibration of circular plates in contact with fluid," Elsevier.

[8] B. Shieh, K. G. Sabra, and F. Levent Degertekin, "A Hybrid Boundary Element Model for Simulation and Optimization of Large Piezoelectric Micromachined Ultrasonic Transducer Arrays," IEEE Trans. Ultrason. Ferroelectr. Freq. Control, vol. 65, no. 1, pp. 50-59, Jan. 2018.

[9] A. Ben Amar, H. Cao, and A. B. Kouki, "Modeling and process design optimization of a piezoelectric micromachined ultrasonic transducers (PMUT) using lumped elements parameters," Microsyst. Technol., vol. 23, no. 10, pp. 4659-4669, Oct. 2017.

\section{CONTACT}

*Boshen Liang, phone: +32-016-283-402;

Email: boshen.liang@imec.be 